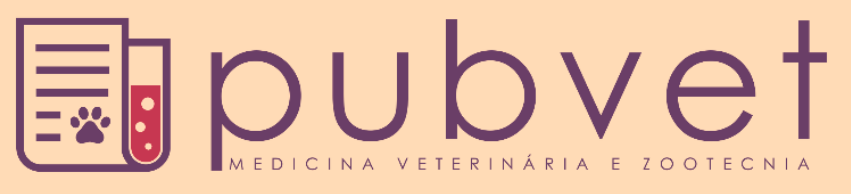

https://doi.org/10.31533/pubvet.v15n12a993.1-5

\title{
Excisão cirúrgica de abscesso em coelho (Oryctolagus cuniculus): Relato de caso ${ }^{\circ}$
}

\author{
Stephane de Paula Santos ${ }^{1 *}$, Larissa Bessa Reis ${ }^{1}$, Thiago Francisco Costa $\operatorname{Solak}^{20}$, \\ Sharlenne Leite da Silva Monteiro $^{30}$, Gabriela Basilio Roberto ${ }^{30}$ \\ ${ }^{I}$ Acadêmica da Universidade Estadual do Centro-Oeste, Guarapuava-PR Brasil. \\ ${ }^{2}$ Especializado em Medicina de Animais Selvagens e Residente da Universidade Federal do Paraná - Curitiba - PR Brasil. \\ ${ }^{3}$ Docente da Universidade Estadual do Centro-Oeste, Departamento de Medicina Veterinária, Guarapuava -PR Brasil. \\ *Autor para correspondência.E-mail: stephanemedvet@gmail.com
}

Resumo. Um coelho (Oryctolagus cuniculus), macho, adulto, pesando $3,3 \mathrm{~kg}$, apresentando uma massa de aspecto firme localizada na região torácica ventral, feridas aparentes de mordedura na mesma região e histórico de briga recente com outro coelho, recebeu atendimento médico veterinário. A suspeita clínica inicial foi abscesso subcutâneo, baseada na anamnese e exame físico, e a mesma foi confirmada através do exame citológico. O tratamento realizado foi cirúrgico, utilizando a técnica de marsupialização. Após dez dias de pós-operatório, constatou que esse tratamento não obteve resultados satisfatórios, pois não houve redução do abscesso. Visto isso, foi optado por realizar excisão cirúrgica do abscesso. Esse segundo tratamento teve resultados satisfatórios, pois foi possível a remoção completa do abscesso e não houve recidiva. Paciente recebeu alta após quinze dias. Dessa forma, conclui-se com base na literatura e nas observações feitas a partir do caso descrito que o recurso terapêutico mais indicado para o tratamento de abscessos subcutâneos em coelhos é a excisão cirúrgica. A cirurgia de marsupialização de abscesso não se mostrou efetiva devido extensão e localização do abscesso, sendo essa técnica recomendada para casos em que o abscesso está profundamente ligado a estruturas subcutâneas e não é possível sua excisão completa e quando se encontra localizado na região do períneo ou na base da orelha, caso contrário, há grandes chances de recidiva e falha no tratamento.

Palavras chave: Anestesia, antibioticoterapia, cirurgia, marsupialização

\section{Surgical excision of abscess in rabbit (Oryctolagus cuniculus): Case report}

Abstract. An adult male rabbit (Oryctolagus cuniculus), weighing $3.3 \mathrm{~kg}$, presenting a firm looking mass located in the ventral thoracic region, apparent bite wounds in the same region and history of recent fight with another rabbit, received veterinary medical attention. The initial clinical suspicion was subcutaneous abscess, based on the anamnesis and physical examination, and it was confirmed through cytological examination. The treatment was surgical, using the marsupialization technique. After ten days postoperatively, it was found that this treatment did not achieve satisfactory results, because there was no reduction of the abscess. Therefore, it was decided to perform a surgical excision of the abscess. This second treatment had satisfactory results, as it was possible to completely remove the abscess and there was no recurrence. The patient was discharged after fifteen days. Based on the literature and our observations of the case described here, we conclude that surgical excision is the most suitable treatment for subcutaneous abscesses in rabbits. The abscess marsupialization surgery was not effective due to the extent and location of the abscess, and this technique is recommended for cases in which the abscess is deeply attached to subcutaneous structures and its complete excision is not possible, and when it is located in 
the perineal region or at the base of the ear, otherwise there are great chances of recurrence and treatment failure.

Keywords: Anesthesia, antibiotic therapy, marsupialization, surgery

\section{Introdução}

Abscessos são coleções localizadas de tecido inflamatório purulento produzidos pela inoculação de bactérias piogênicas dentro de um tecido (Oliveira, 2012). Em coelhos, abscessos subcutâneos são comuns e podem ser resultantes de feridas traumáticas ou bacteremia secundária (Carpenter, 2007), uma vez que a espécie possui uma epiderme fina e sem aderência de colágeno entre a derme e as camadas mais profundas (Harcourt-Brown \& Chitty, 2013), resultando em uma pele extremamente fina e fácil de ser lesionada (Bennett Junior, 2011). Nessa espécie, os abscessos normalmente possuem parede espessa e o exsudato é muito viscoso e tenaz devido falta da enzima mieloperoxidase nos heterófilos, que possui função de liquefazer o pus. Devido a essas características os abscessos em coelhos podem ser difíceis de tratar. Os antibióticos sistêmicos não conseguem penetrar a cápsula e a simples punção e lavagem geralmente não é eficaz, a melhor opção é a excisão cirúrgica (Flecknell, 2008), mas isso vai depender da localização e extensão do abscesso. O diagnóstico é confirmado por exame citológico (Carpenter, 2007). Devido à escassez de trabalhos publicados referente a área e espécie em questão, esse trabalho tem como objetivo relatar e comparar a eficácia de duas diferentes técnicas cirúrgicas utilizadas para tratamento de abscesso subcutâneo em coelhos e destacar a importância do tratamento cirúrgico nesses casos, que devido as características do abscesso nessa espécie, torna difícil e até mesmo impossível a solução do caso somente com tratamento clínico.

\section{Relato do caso clínico}

Um coelho (Oryctolagus cuniculus), macho, adulto, pesando $3,3 \mathrm{~kg}$, apresentando uma massa de aspecto firme localizada na região torácica ventral, feridas aparentes de mordedura na mesma região e histórico de briga recente com outro coelho, recebeu atendimento médico veterinário. A suspeita clínica inicial foi abscesso subcutâneo, baseada na anamnese e exame físico, e a mesma foi confirmada através do exame citológico.

O tratamento realizado foi cirúrgico, utilizando a técnica de marsupialização. O protocolo anestésico escolhido foi cloridrato de tramadol $(5 \mathrm{mg} / \mathrm{kg}$, v.o) como medicação pré-anestésica e isoflurano para indução e manutenção anestésica no vaporizador universal. Para fluidoterapia foi utilizado ringer com lactato i.v. na veia marginal da orelha. No pós-cirúrgico imediato foi administrado meloxicam $(0,2$ $\mathrm{mg} / \mathrm{kg}$, i.v) e enrofloxacina ( $5 \mathrm{mg} / \mathrm{kg}$, i.v). Na sequência foi administrado meloxicam $(0,2 \mathrm{mg} / \mathrm{kg}$, v.o, SID, por 1 dia) e enrofloxacina ( $5 \mathrm{mg} / \mathrm{kg}$, v.o, BID, por 10 dias), probiótico ( $2 \mathrm{~g} /$ dia, v.o, BID, durante 10 dias), cloridrato de metoclopramida ( $5 \mathrm{mg} / \mathrm{kg}$, v.o, BID, durante 2 dias) e cloridrato de tramadol (5 $\mathrm{mg} / \mathrm{kg}$, v.o, BID, durante 3 dias). No tratamento tópico foi realizado limpeza com solução fisiológica e aplicado spray de rifamicina sódica TID, por 10 dias) e pomada de sulfato de neomicina com bacitracina zíncica (TID, por 10 dias).

Após dez dias, esse tratamento não obteve resultados satisfatórios, pois não houve redução do abscesso. Visto isso, foi optado por realizar excisão cirúrgica do abscesso. O protocolo anestésico utilizado foi o mesmo da primeira cirurgia. No pós-cirúrgico imediato foi administrado enrofloxacina $(5 \mathrm{mg} / \mathrm{kg}$, i.v) e meloxicam $(0,2 \mathrm{mg} / \mathrm{kg}$, i.v). Na sequência foi administrado enrofloxacina $5 \mathrm{mg} / \mathrm{kg}$, v.o, BID, por 7 dias) e probiótico (2 g/dia, v.o, BID, durante 7 dias), tramadol (5 mg/kg, v.o, BID, durante 3 dias), metoclopramida (5 mg/kg, v.o, BID, durante 3 dias e meloxicam $(0,2 \mathrm{mg} / \mathrm{kg}$, v.o, SID, por 2 dias), além do tratamento tópico da ferida cirúrgica com solução fisiológica e aplicação de rifamicina sódica (TID, por 15 dias). Esse segundo tratamento teve resultados satisfatórios, pois foi possível a remoção completa do abscesso e não houve recidiva. Paciente recebeu alta após 15 dias.

\section{Discussão}

O paciente chegou à clínica com o histórico de briga recente com outro coelho, apresentando também uma massa de aspecto firme em região torácica ventral. A suspeita clínica mais comum nesses casos é a formação de abscesso, uma vez que a flora bucal do outro coelho envolvido na briga e alguns 
microorganismos ambientais têm potencial piogênico ao serem inoculados no tecido íntegro. É improvável a resolução natural dos abscessos nesta espécie, pois, entre outros fatores, a atuação das células de defesa é atenuada pela característica do líquido com restos necróticos e $\mathrm{pH}$ muito baixo. Da mesma forma, o simples tratamento com antibioticoterapia também não seria eficaz na resolução do caso, uma vez que os fármacos não conseguiriam penetrar na cápsula inflamatória, não conseguindo assim, atingir o centro necrótico (Oliveira, 2012). Por esse motivo, a fim de evitar disseminação bacteriana e aliviar o incômodo trazido pelas alterações locais, que causam compressão e dor, a intervenção cirúrgica (Figura 1) foi a terapêutica indicada para o caso após a confirmação da suspeita clínica através do exame citológico.
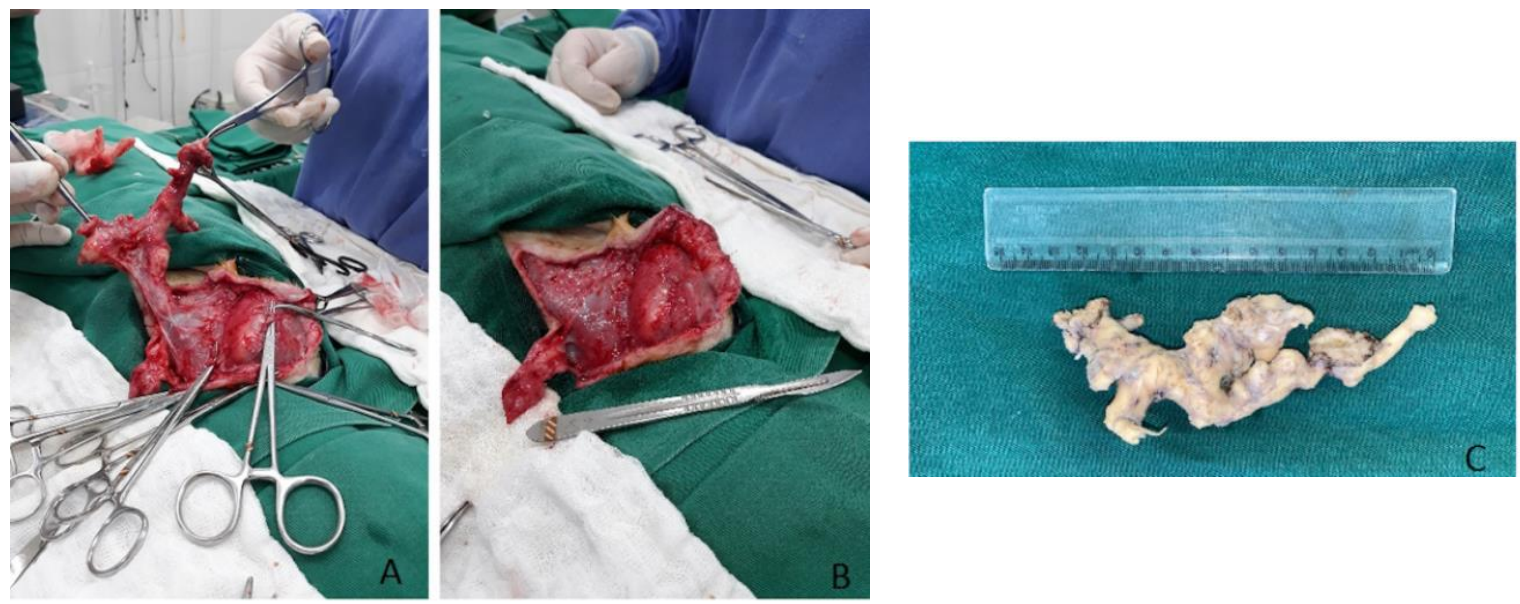

Figura 1. Imagem fotográfica do trans-operatório (A) e (B) e a imagem do abscesso já formalizado em (C). Em (A) pode-se observar a dissecação do abscesso mantendo a integridade da cápsula para evitar a contaminação do campo cirúrgico. Já em (B) nota-se a completa remoção do abscesso e os tecidos adjacentes limpos e livres de contaminação. Aproximou-se um bisturi para um comparativo do tamanho da incisão cirúrgica. Por último em (C) nota-se o abscesso já formalizado ao lado de uma régua para avaliar seu tamanho real.

Apesar da inflamação aguda em resposta à infecção supurativa, não foram observados no paciente sinais sistêmicos de inflamação, como dor e febre, sendo essa uma particularidade comum da espécie (Harcourt-Brown \& Harcourt-Brown, 2002). A aparente ausência de sinais de dor apresentada pelo paciente antes da cirurgia pode ser justificada pela característica da espécie de mascarar ao extremo esses sinais. Dessa forma, Fantoni \& Mastrocinque (2005) sugerem que a analgesia preventiva deve ser sempre feita a fim de se certificar que o controle de dor está sendo eficaz.

O protocolo medicamentoso utilizado para medicação pré-anestésica foi cloridrato de tramadol (5 $\mathrm{mg} / \mathrm{kg}$, v.o) pois é indicado para tratar dor aguda moderada a severa (Carpenter, 2010). A indução e manutenção anestésica foram realizadas utilizando isoflurano com uma máscara facial, foi escolhido realizar anestesia inalatória com isoflurano pois oferece uma indução e recuperação rápidas e a capacidade de alterar rapidamente a profundidade anestésica (Carroll, 2012; Fantoni et al., 2002; Quesenberry \& Carpenter, 2011), além de promover moderada analgesia e relaxamento muscular (Carroll, 2012; Fantoni et al., 2002; Quesenberry \& Carpenter, 2011). Durante o procedimento anestésico o paciente apresentou parâmetros dentro dos valores de referência para a espécie, frequência cardíaca de 150 a 300 batimentos por minuto (Cracknell, 2008), frequência respiratória de 30 a 60 movimentos por minuto (Cracknell, 2008; Flecknell, 2008; Wenger, 2012), temperatura corporal de 38,5 a $40^{\circ} \mathrm{C}$ (Cracknell, 2008; Flecknell, 2008; Wenger, 2012). Durante todo o procedimento anestésico foi fornecido suporte de oxigênio $100 \%$ com uma máscara facial e fluidoterapia aquecida utilizando ringer com lactato i.v. pela veia marginal da orelha, uma vez que a espécie é suscetível às complicações trans cirúrgicas como desidratação, hipotermia, choque hipovolêmico, depressão respiratória e renal (Harkness et al., 2010).

O paciente foi anestesiado e posicionado em decúbito dorsal, de modo que a área em que se localizava o abscesso fosse facilmente acessível ao cirurgião. Foi realizada tricotomia com ampla margem para permitir a remoção do abscesso e posteriormente a antissepsia da pele com iodo povidine (HarcourtBrown \& Chitty, 2013; Quesenberry \& Carpenter, 2011). 
Foi realizada a incisão da pele com bisturi ao redor do abscesso e divulsionados os tecidos com uma tesoura metzembaum para poder remover o abscesso, com muita cautela pois em certos pontos a cápsula pode estar aderida e o manuseio pode causar sua ruptura (Harcourt-Brown \& Chitty, 2013; Quesenberry \& Carpenter, 2011). Nos pequenos vasos foi realizado ligadura com fio absorvível sintético de ácido poliglicólico de diâmetro 3/0. Após a retirada do abscesso, foi feita lavagem da região com solução fisiológica estéril e posteriormente a sutura de subcutâneo e da pele. Para a sutura de subcutâneo foi utilizado o padrão de subcutâneo, utilizando fio absorvível sintético de ácido poliglicólico de diâmetro 3/0 e para a pele foi realizada sutura simples interrompida, utilizando fio inabsorvível de nylon de diâmetro 3/0 (Harcourt-Brown \& Chitty, 2013; Quesenberry \& Carpenter, 2011).

No pós-operatório imediato foi administrado enrofloxacina (5 mg/kg i.v) (Carpenter, 2007) e meloxicam (0,2 mg/kg i.v) (Carpenter, 2007; Lichtenberger, 2005; Longley, 2008). A antibioticoterapia no pós-operatório deve ser feita com muita cautela, principalmente com o uso de beta lactâmicos, macrolídeos e lincosamidas, pois irá alterar a flora entérica normal, podendo levar à um quadro de enterotoxemia com relatos de mortes de pacientes a partir de 48 horas do início do tratamento (Harkness et al., 2010). Foi utilizado como medicação pós-operatória probiótico (2 g/dia, v.o, BID, por 7 dias) e enrofloxacina (5 mg/kg, v.o, BID, por 7 dias) (Carpenter, 2007), contradizendo Saunders \& Davies (2005) que não consideram uma boa escolha o uso desse antibiótico para feridas e abscessos causados por mordidas, pois não tem efeito contra bactérias anaeróbicas. Para controle da dor foi utilizado meloxicam (0,2 mg/kg, v.o, BID, por 2 dias) (Carpenter, 2007; Lichtenberger, 2005; Longley, 2008) associado ao cloridrato de tramadol ( $5 \mathrm{mg} / \mathrm{kg}$, v.o, BID) para controle da dor (Carpenter, 2007), contradizendo Lichtenberger (2005), que recomenda a dose 2 a $4 \mathrm{mg} / \mathrm{kg}$, v.o, BID para tramadol, essa associação fornece uma analgesia adequada para cirurgias de retirada de abscesso em coelhos (HarcourtBrown \& Chitty, 2013; Harcourt-Brown \& Harcourt-Brown, 2002). Como os opioides podem causar efeitos adversos significativos nos mamíferos, incluindo a diminuição da motilidade gastrointestinal (Paul-Murphy, 2007), a metoclopramida $(0,5 \mathrm{mg} / \mathrm{kg}$, v.o., BID, durante três dias) foi administrada profilaticamente (Carpenter, 2007; Harcourt-Brown \& Harcourt-Brown, 2002; Lichtenberger, 2005; Longley, 2008; Saunders \& Davies, 2005). Para tratamento tópico da ferida cirúrgica foi utilizado solução fisiológica e aplicação de rifamicina sódica por 15 dias.

No pós-operatório o paciente não apresentou complicações. Após 15 dias foi realizada a retirada dos pontos da pele e o paciente recebeu alta (Figura 2). A excisão cirúrgica do abscesso se mostrou eficaz e o paciente não apresentou recidiva.

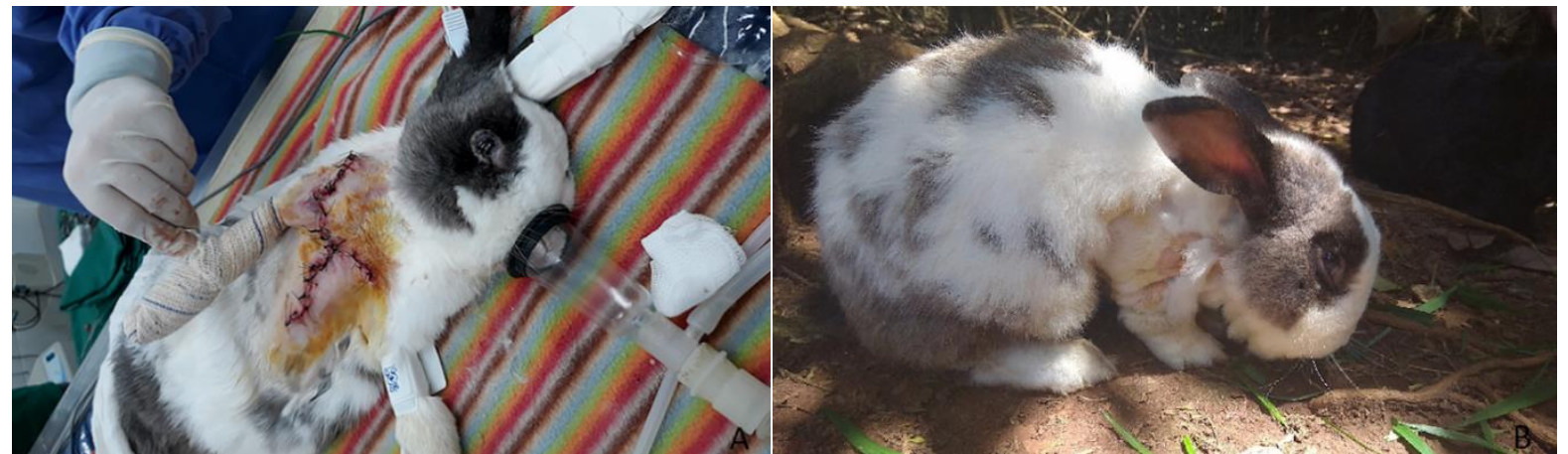

Figura 2. Imagem fotográfica comparativa do pós-operatório imediato (A) e pós-operatório tardio (B). Em (A) pode-se observar a ferida cirúrgica após a remoção completa do abscesso. Também a presença do acesso venoso na veia marginal da orelha e o uso da máscara para manutenção da anestesia inalatória com isoflurano. No membro torácico esquerdo observa-se o manguito para mensuração da pressão sanguínea durante a anestesia do animal. Em (B) observa-se o animal já em casa com completa recuperação e cicatrização com início de crescimento dos pelos na área de tricotomia.

Dessa forma, conclui-se com base na literatura e nas observações feitas a partir do caso descrito que o recurso terapêutico mais indicado para o tratamento de abscessos subcutâneos em coelhos é a excisão cirúrgica. A cirurgia de marsupialização de abscesso não se mostrou efetiva devido extensão e localização do abscesso, assim já relatada ineficácia em casos com essas características por Vargas, (2013), essa técnica é recomendada para casos em que o abscesso está profundamente ligado a estruturas subcutâneas e não é possível sua excisão completa e quando se encontra localizado na região do períneo ou na base da orelha, caso contrário, há grandes chances de recidiva e falha no tratamento. 


\section{Referências bibliográficas}

Bennett Junior, R. A. (2011). General principles of surgical techniques. In J W Carpenter, K. Quesenberry, \& O. C. Mans (Eds.), Clinical medicine and surgery. Elsevier Health Sciences. https://doi.org/10.1016/C2015-0-05982-2.

Carpenter, J W. (2007). Exotic animal formulary. 3.ed.St. Sauders: Elsevier, Louis, Missouri, USA. Saunders Elsevier.

Carpenter, James W. (2010). Formulário de animais exóticos. MedVet Livros.

Carroll, G. L. (2012). Anestesia e analgesia de pequenos animais (Manole (ed.); Vol. 6460).

Cracknell, J. (2008). Anaesthesia of exotic pets. The Veterinary Record, 162(26), 864.

Fantoni, D. T., \& Mastrocinque, S. (2005). Analgesia preventiva. In P. E. Otero (Ed.), Dor: Avaliação e tratamento em pequenos animais (pp. 76-80). Interbook.

Fantoni, D. T., Mastrocinque, S., Fantoni, D. T., \& Cottopassi, S. R. (2002). Fisiopatologia e controle da dor. In D. T. Fabntoni (Ed.), Anestesia em cães e gatos (pp. 323-336). Roca.

Flecknell, P. A. (2008). Analgesia from a veterinary perspective. British Journal of Anaesthesia, 101(1), 121-124. https://doi.org/https://doi.org/10.1093/bja/aen087.

Harcourt-Brown, F., \& Chitty, J. (2013). BSAVA manual of rabbit surgery, dentistry and imaging. In BSAVA Manual of rabbit surgery, dentistry and imaging. British Small Animal Veterinary Association.

Harcourt-Brown, F., \& Harcourt-Brown, N. H. (2002). Textbook of rabbit medicine. ButterworthHeinemann.

Harkness, J. E., Turner, P. V, VandeWoude, S., \& Wheler, C. L. (2010). Harkness and Wagner's biology and medicine of rabbits and rodents. John Wiley \& Sons.

Lichtenberger, M. (2005). Fluid resuscitation and nutritional support in rabbits with gastric stasis or gastrointestinal obstruction. Exotic DVM, 7(2), 34-38.

Longley, L. (2008). Rodent anaesthesia. Anaesthesia of Exotic Pets, 59-84.

Oliveira, A. L. A. (2012). Técnicas cirúrgicas em pequenos animais. Elsevier Brasil.

Paul-Murphy, J. (2007). Critical care of the rabbit. Veterinary Clinics of North America: Exotic Animal Practice, 10(2), 437-461.

Quesenberry, K., \& Carpenter, J. W. (2011). Ferrets, rabbits and rodents: clinical medicine and surgery. Elsevier Health Sciences.

Saunders, R. A., \& Davies, R. R. (2005). Notes on rabbit internal medicine. Black Acaddemic \& Professional.

Vargas M. (2013). Basic principles of soft tissue surgery. In: Chitty J., Harcourt-Brown F. (Eds). BSAVA manual of rabbit surgery, dentistry and imaging. Quedgeley:British Small Animal Veterinary Association.

Wenger, S. (2012). Anesthesia and analgesia in rabbits and rodents. Journal of Exotic Pet Medicine, $21(1), 7-16$.

Histórico do artigo:

Recebido: 21 de julho de 2021

Aprovado: 2 de setembro de 2021

Disponível on-line: 12 de novembrode 2021
Licenciamento: Este artigo é publicado na modalidade Acesso Aberto sob a licença Creative Commons Atribuição 4.0 (CC-BY 4.0), a qual permite uso irrestrito, distribuição, reprodução em qualquer meio, desde que o autor e a fonte sejam devidamente creditados. 